\title{
PREFACE
}

\section{Stepped surfaces}

To cite this article: Ludo Juurlink 2018 J. Phys.: Condens. Matter 30090301

View the article online for updates and enhancements.

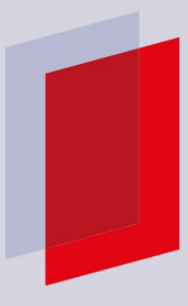

\section{IOP ebooks}

Bringing you innovative digital publishing with leading voices

to create your essential collection of books in STEM research.

Start exploring the collection - download the first chapter of every title for free. 


\section{Preface}

\section{Stepped surfaces}

\section{Ludo Juurlink (1)}

Leiden Institute of Chemistry, Leiden University, PO

Box 9502, 2300 RA Leiden, Netherlands

E-mail: 1.juurlink@chem. leidenuniv.nl
The explanation of spot splitting in low energy electron diffraction (LEED) patterns of surfaces vicinal to low Miller-index planes by Ellis and Schwoebel [1] and Henzler [2] opened the doors to studying how atomic steps influence adsorption of atoms and molecules. The earliest papers discussing such topics for macroscopically flat, but microscopically highly corrugated surfaces, thus stem from the early 1970s, adsorption and reaction initially being studied by LEED [3], flash desorption [4] and molecular beam techniques [5]. This special issue on Adsorption Phenomena on Stepped Surfaces starts with a historical perspective by Woodruff from Warwick University [6]—one of the first scientists who also explored the use of curved single crystals to probe the effects of steps on adsorption and reaction [7].

One of the most widely accepted ideas resulting from several decades of research on stepped surfaces is that the low-coordinated atoms in the step have an increased ability to dissociate adsorbing molecules. The origin of this effect is generally explained by an increased binding energy of the fragments as compared to the low Miller plane. Nørskov et al showed 15 years ago that scaling relations between the adsorption energy of dissociated diatomics and the activation barrier for dissociative adsorption also hold on stepped surfaces [8]. Defect sites on catalytic particles, which strongly resemble steps on single crystals, turn out to be crucial to various industrial catalytic processes

$[9,10]$. They may also be important to processes that ultimately change the selvage or bulk of the material, e.g. in bulk oxidation or metal-hydride formation. This specal issue includes a paper by Farber et al that investigates to what extent step defects induce absorption of $\mathrm{O}$ atoms into the selvedge of $\mathrm{Pt}[11]$.

The energetics of molecular adsorption does, on the other hand, not always differ much for adsorption to steps and terraces. An example is provided in this issue in a combined density functional theory (DFT) and temperature programmed desorption study for CO adsorbing to $\mathrm{Cu}$ surfaces with (100) planes by Kokalj et al [12]. Recently, a similar combined experimental and theoretical reaction dynamics study also showed that steps can even lower reactivity- $\mathrm{Cu}(211)$ was shown to be less reactive toward hydrogen dissociation than $\mathrm{Cu}\left(\begin{array}{lll}1 & 1 & 1\end{array}\right)$ [13].

The adsorption of larger molecules on steps has also attracted considerable interest. For tri-atomics, $\mathrm{H}_{2} \mathrm{O}$ stands out as the binding and reaction of water with corrugated metal interfaces is highly relevant to, e.g. electrochemistry [14]. Steps influence the adsorption of water, often by inducing adsorption structures deviating rather drastically from the molecular ordering in bulk hexagonal ice, $I_{\mathrm{h}}$. Steps can induce one-dimensional chain formation along edges with uncommon structural features on $\mathrm{Pt}$ [15], 2D patterning that includes pentamers, hexamers and octamers on $\mathrm{Cu}[16]$ and have even been shown to be the origin of growth of uncommon $3 \mathrm{D}$ cubic ice, $I_{\mathrm{C}}[17]$.

This special issue on Adsorption Phenomena on Stepped Surfaces provides two examples of studies investigating much larger molecules. Gellman et al use the inherent chirality of kinked-stepped surfaces and beautifully show how small variations in the interaction with a chiral cyclohexanone-derivative depends on more than chirality alone [18]. Jiang et al investigate the influence of benzene adsorption on work function changes [19], showing how theoretical treatment is progressing by including van der Waals interactions in their DFT study and using a range of Ag stepped surfaces extending up to seven atom-wide terraces.

The contributions in the issue show that, whereas nearly five decades of research has taught us much on how steps alter the properties of surfaces, there is still much to learn and discover. For that reason the recent revival of the use of curved samples is of high interest. With similar sizes as regular flat samples, the curved ones provide step density variations that may span up to three orders of magnitude while remaining suitable for study in many 
laboratories. They have recently proven their use in studies of physical aspects, e.g. mapping of surface electronic state dependencies on stepped surface structure [20], and chemical aspects, e.g. adsorption and desorption of $\mathrm{CO}[21,22]$ and $\mathrm{H}_{2} \mathrm{O}$ [23], and chemical reactivity for elementary dissociative reactions [24] and overall reactivity [25].

\section{ORCID iDs}

Ludo Juurlink (i) https://orcid.org/0000-0002-5373-9859

\section{References}

[1] Ellis W P and Schwoebel R L 1968 Surf. Sci. 11 82-98

[2] Henzler M 1970 Surf. Sci. 19 159-71

[3] Lang B et al 1972 Surf. Sci. 30 454-74

[4] Lu K E and Rye R R 1974 Surf. Sci. 45 677-95

[5] Bernasek S L and Somorjai G A 1975 J. Chem. Phys. 62 3149-61

[6] Woodruff D P 2016 J. Phys.: Condens. Matter 28491001

[7] Armitage A F et al 1980 Surf. Sci. 100 L483-90

[8] Nørksov J K et al 2002 J. Catal. 209 275-8

[9] Honkala K et al 2005 Science 307 555-8

[10] Behrens M et al 2012 Science 336 893-7

[11] Farber R G et al 2017 J. Phys.: Condens. Matter 29164002

[12] Kokalj A et al 2017 J. Phys.: Condens. Matter 29194001

[13] Füchsel G 2018 J. Phys. Chem. Lett. 9 170-5

[14] van der Niet M J T C et al 2013 Catal. Today 202 105-13

[15] Kolb M J et al 2016 Phys. Rev. Lett. 116136101

[16] Lin C et al 2018 Phys. Rev. Lett. accepted (https://journals.aps.org/prl/accepted/4007aY51X241c 15909591d13a4dd51ce098ba7135)

[17] Thurmer K and Nie S 2013 Proc. Natl Acad. Sci. 110 11757-62

[18] Gellman A J et al 2017 J. Phys.: Condens. Matter 29034001

[19] Jiang Y et al 2017 J. Phys.: Condens. Matter 29204001

[20] Ortega J E et al 2011 Phys. Rev. B 83085411

[21] Walsh A J et al 2017 J. Vac. Sci. Technol. A 3503 E102

[22] Walter A L et al 2015 Nat. Commun. 68903

[23] Janlamool J et al 2014 Molecules 19 10845-62

[24] Hahn C et al 2012 J. Chem. Phys. 136114201

[25] Blomberg S et al 2017 ACS Catal. 7 110-4 